\title{
Inverse Anti- $k$-centrum Problem on Networks with Variable Edge Lengths
}

\author{
Van Huy Pham and Kien Trung Nguyen*
}

\begin{abstract}
This paper concerns the problem of modifying edge lengths of a network at minimum total costs so as to make a prespecified vertex become an optimal location in the modified environment. Here, we focus on the ordered median objective function with respect to the vector of multipliers $\lambda=(1, \ldots, 1,0, \ldots, 0)$ with $k 1$ 's. This problem is called the inverse anti- $k$-centrum problem. We first show that the inverse anti- $k$-centrum problem is NP-hard even on tree networks. However, for the inverse anti- $k$-centrum problem on cycles, we formulate it as one or two linear programs, depending on odd or even integer $k$. Concerning the special cases with $k=2,3, M$, we develop combinatorial algorithms that efficiently solve the problem, where $M$ is the number of vertices of the cycle.
\end{abstract}

\section{Introduction}

The location problem plays an important role in operations research due to its prominent real-life applications. In this problem, we aim to find optimal locations of new facilities. For the detailed survey of location problems, one can refer to [12,13, 18,. While the classical location problem often involves the median or center objective function, the so-called ordered median problem is considered with the unified objective function, a generalization of a class of functions. For references on the ordered median problem, one can see the book of Nickel and Puerto [25].

Recently, the inverse location problem has become an interesting research topic. Here, we aim to modify parameters, vertex weights or edge lengths, at minimum total cost so that prespecified locations becomes optimal with respect to new parameters. Inverse median and inverse center problems are the two popular models among the classical inverse location problems. In the following let us review literatures concerning the inverse median and center problems together with their complexity depending on the input data size $M$.

The inverse median problem was first investigated by Burkard et al. [9], where they solved the inverse 1 -median problem on trees and on the plane with $l_{1}$-norm in $O(M \log M)$

Received December 4, 2018; Accepted June 13, 2019.

Communicated by Jein-Shan Chen.

2010 Mathematics Subject Classification. 90B10, 90B80, 90C27.

Key words and phrases. location problems, inverse optimization problems, ordered median function, anti$k$-centrum, tree, cycle.

${ }^{*}$ Corresponding author. 
time. Then Galavii [14] improved the complexity for solving the inverse 1-median problem on trees to linear time. Also, Nguyen [20] considered the inverse 1-median problem on a generalization of tree graphs, the so-called block graphs. Based on the convexity of the cost function, he solved this problem in $O(M \log M)$ time. Furthermore, Burkard et al. [10] solved the inverse 1-median problem on cycles with variable vertex weights in $O\left(M^{2}\right)$ time by exploring the concavity of the corresponding linear programming constraints. Bonab et al. 7 considered the inverse $p$-median problem on networks. It is shown that the problem on general networks is NP-hard. However, the inverse 2-median problem on trees is solvable in polynomial time. Additionally, if the underlying tree is a star, then the inverse 2-median problem can be solved in linear time. The inverse 1-median problem on the plane was also widely investigated. Burkard et al. 8 solved the inverse FermatWeber problem with variable facility weights by a combinatorial algorithm in $O(M \log M)$ time. The inverse 1-median problem with variable coordinates was investigated by Bonab et al. [6]. They solved the problem on $\mathbb{R}^{d}$ with $l_{2}^{2}$-norm in $O(M d)$ time. However, the problem with $l_{1}$ - or $l_{\infty}$-norm is NP-hard.

Concerning the inverse 1-center problem, Cai et al. 11] showed that although the 1center problem on directed networks can be solved in polynomial time, the inverse version is NP-hard. Therefore, it is interesting to further study some special situations of inverse 1-center problem on networks which are polynomial solvable. Alizadeh, Burkard, and Pferschy [2,3,5] developed a combinatorial algorithms that solve the inverse 1-center problem on trees under $l_{1}$-norm. Moreover, Nguyen and Sepasian [24] investigated this problem under Chebyshev norm and bottleneck Hamming distance. Based on the special structure of the objective function, they showed that the problem can be solved in quadratic time for both cases, with or without topology changes.

The inverse convex ordered 1-median problem on trees with variable edge lengths was investigated by Gassner [16. She showed that both the inverse convex ordered 1-median on unweighted trees and the inverse $k$-centrum problem on weighted trees under rectilinear norm are NP-hard. However, the inverse $k$-centrum problem on an unweighted tree can be solved in $O\left(M^{2} k^{3}\right)$ time by dynamic programming. For some polynomially solvable cases of this problem, one can see [21]. Concerning the inverse convex ordered 1-median problem under Chebyshev norm, Nguyen and Chassein 23 developed $O\left(M^{2} \log M\right)$ algorithm based on the greedy technique and binary search. Furthermore, the inverse $k$-centrum problem on trees with variable weights is NP-hard; see Nguyen and Anh 22. They also develop a quadratic algorithmic for the inverse 1-center problem, a special case with $k=1$.

Existing models for inverse ordered 1-median problem on networks addressed the convex case, i.e., the multipliers are nondecreasing and the underlying network is a tree. Problem with other classes of multipliers or on cycle networks is, however, not under 
investigated so far. We consider in this paper the inverse anti- $k$-centrum problem on networks, in which the $k$ smallest weighted distances to a specified vertex contribute to the objective function. We organize the paper as follows. We recall in Section 2 some preliminary definitions concerning the ordered 1-median function and the inverse ordered 1 -median problem. Section 3 shows the NP-hardness of the inverse anti- $k$-centrum problem on trees. In Section 4, we prove that the inverse anti- $k$-centrum problem on an unweighted cycle can be solve in polynomial time. Furthermore, the special cases $k=2,3$ are addressed with efficient combinatorial algorithms. The inverse 1-median problem on unweighted cycles with variable edge lengths, a special case of the inverse anti- $k$-centrum problem with $k=M$, is solvable in quadratic time, provided that the edge lengths are only allowed to be increased or decreased.

\section{Preliminaries and problem formulation}

We first recall the concept of ordered 1-median function on networks; see [25]. Given a connected graph $G=(V, E), V=\left\{v_{1}, \ldots, v_{M}\right\}$, each vertex $v_{i} \in V$ is associated with a nonnegative weight $w_{i}$ and each edge $e \in E$ has a nonnegative length $\ell_{e}$. The distance $d(u, v)$ between two vertices $u$ and $v$ in $G$ is the length of the shortest path connecting these two vertices. Here, the shortest path connecting $u$ and $v$ is the one such that the sum of the lengths of its constituent edges is minimized. A point $\rho$ lies on $G$ if it lies on some edge $e=(u, v) \in G$ and identified by choosing $t \in[0,1]$ such that $d(u, \rho)=t \ell_{e}$. It is obviously observe that $\rho=u$ if $t=0$ and $\rho=v$ if $t=1$. We denote the set of all points on $G$ by $A(G)$. The distance between two points on $G$ is defined similarly to that of two vertices. Given a vector of multipliers $\lambda=\left(\lambda_{1}, \lambda_{2}, \ldots, \lambda_{M}\right) \in \mathbb{R}_{+}^{M}$, the ordered 1-median objective function defined on $A(G)$ is

$$
f_{\lambda}(\rho)=\sum_{i=1}^{M} \lambda_{i} w_{(i)} d\left(\rho, v_{(i)}\right)
$$

for $\rho \in A(G)$. Here, operator $(\cdot)$ is a permutation on the index set of vertices of $G$ satisfying

$$
w_{(1)} d\left(x, v_{(1)}\right) \leq w_{(2)} d\left(x, v_{(2)}\right) \leq \cdots \leq w_{(M)} d\left(x, v_{(M)}\right) .
$$

A point $\rho^{*}$ is called an ordered 1-median of $G$ if and only if $f_{\lambda}\left(\rho^{*}\right) \leq f_{\lambda}(\rho)$ for all $\rho \in A(G)$. If the multipliers satisfy $\lambda_{1} \geq \lambda_{2} \geq \cdots \geq \lambda_{M}$, the ordered 1-median function is concave on each edge of the network. For the concave situation, one of the vertex is an ordered 1-median of $G$. Partially, if the vector of multipliers is specified as

$$
\lambda=(\underbrace{1,1, \ldots, 1,1}_{k 1 \text { 's }}, 0,0, \ldots, 0,0),
$$


the corresponding problem is called the anti- $k$-centrum problem. Once the decision maker interests only on the $k$ clients close to the server, other clients are far away from being influenced, and would like to minimize them, then the anti- $k$-centrum objective function is suitable to model the situation.

Given a connected graph $G=(V, E)$ and a prespecified vertex $v^{*}$ in $V$. The length of each edge $e \in E$ can be increased or decreased by an amount $p_{e}$ or $q_{e}$, respectively. It means the modified length of edge $e$ is $\widetilde{\ell}_{e}:=\ell_{e}+p_{e}-q_{e}$ and it is nonnegative. Furthermore, the cost to increase or decrease one unit length of $e$ is $c_{e}^{+}$or $c_{e}^{-}$. For a vector of multipliers $\lambda \in \mathbb{R}_{+}^{M}$, we denote by $\widetilde{f}_{\lambda}$ the ordered 1 -median with respect to new edge lengths. Let us state the inverse ordered 1-median problem on the given instance as follows.

1. The prespecified vertex $v^{*}$ becomes an ordered 1 -median of $G$, i.e.,

$$
\widetilde{f}_{\lambda}\left(v^{*}\right) \leq \tilde{f}_{\lambda}(\rho)
$$

for all $\rho \in A(G)$.

2. The cost function $\sum_{e \in E}\left(c_{e}^{+} p_{e}+c_{e}^{-} q_{e}\right)$ is minimized.

3. Variables are in certain bounds, i.e., $0 \leq p_{e} \leq \bar{p}_{e}$ and $0 \leq q_{e} \leq \bar{q}_{e}$ for $e \in E$.

3. Complexity result of the inverse anti- $k$-centrum problem on trees

Consider an unweighted tree $T=(V, E)$, i.e., all vertex weights in $T$ are equal to 1 . Furthermore, the vector of multipliers is

$$
\lambda:=(\underbrace{1,1, \ldots, 1,1}_{k 1 \text { 's }}, 0,0, \ldots, 0,0),
$$

i.e., the ordered 1-median function is concaved in edge of the tree. In contrast to the polynomial solvability of the inverse $k$-centrum problem on $T$ (see Gassner [16]), we obtain the following result.

Theorem 3.1. The inverse anti-k-centrum problem on unweighted trees is NP-hard.

Proof. Consider an instance $(I)$ of the partition problem as follows. Given a set $S:=$ $\left\{a_{1}, a_{2}, \ldots, a_{n}\right\} \subset \mathbb{N}$ such that $\sum_{i=1}^{n} a_{i}=2 B$. Does there exist a subset $S^{\prime} \subset S$ satisfying $\sum_{a_{i} \in S^{\prime}} a_{i}=B$ ? The partition problem is NP-hard, see Garey and Johnson [15.

The decision version of the inverse anti- $k$-centrum problem on a tree (InvAntiT) is stated as follows. Given an instance of the inverse anti- $k$-centrum problem on an unweighted tree $T=(V, E)$. Does there exist a modification of edge lengths such that the cost is at most $C$ and the prespecified vertex $v^{*}$ becomes an anti- $k$-centrum of the tree?

Given an instance $(I)$, we construct an instance (InvAntiT) in polynomial time in the following way. 
- The vertex set $V:=\left\{v^{*}, v_{0}\right\} \cup\left\{v_{i}, x_{i}, y_{i}\right\}_{i=1, \ldots, n} \cup\left\{z_{j}\right\}_{j=1, \ldots, 2 n}$.

- The edge set $E:=E_{1} \cup E_{2}$, where

$$
E_{1}:=\left\{\left(v^{*}, v_{0}\right)\right\} \cup\left\{\left(v_{0}, v_{i}\right),\left(v_{0}, x_{i}\right),\left(v_{0}, y_{i}\right)\right\}_{i=1, \ldots, n} \quad \text { and } \quad E_{2}:=\left\{\left(v^{*}, z_{j}\right)\right\}_{j=1, \ldots, 2 n} .
$$

- The edge lengths are $\ell_{\left(v^{*}, v_{0}\right)}:=2 B, \ell_{\left(v_{0}, v_{i}\right)}:=B-a_{i}, \ell_{\left(v_{0}, x_{i}\right)}:=B, \ell_{\left(v_{0}, y_{i}\right)}:=2 B$ for $i=1, \ldots, n$, and $\ell_{\left(v^{*}, z_{j}\right)}:=\frac{n+1}{2 n} B$ for $j=1, \ldots, 2 n$.

- The length of $\left(v_{0}, v_{i}\right)$ can be increased by an amount $p_{i}$, with $0 \leq p_{i} \leq 2 a_{i}$, for $i=1, \ldots, n$ and other edge lengths are fixed. The cost to modify one unit edge length is equal to 1 .

- Choose $C:=2 B$ and $k:=3 n+2$.

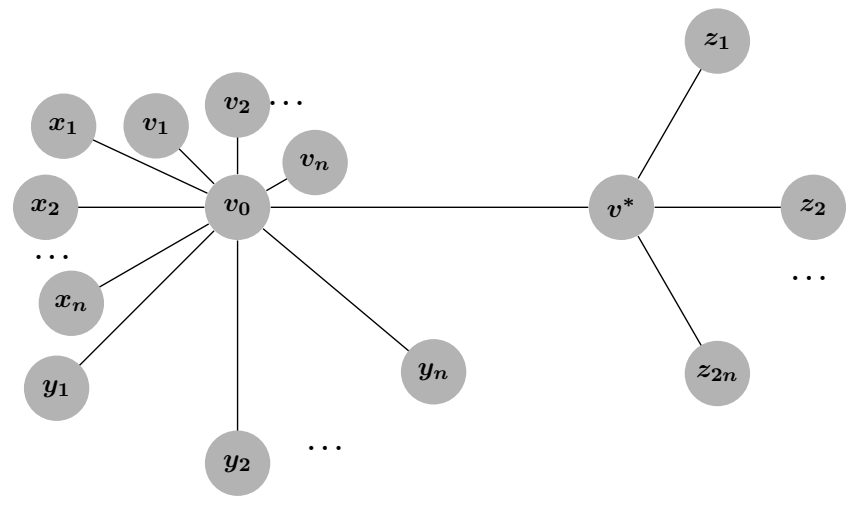

Figure 3.1: The constructed tree $T$ of the instance (InvAntiT).

We consider the inverse anti- $(3 n+2)$-centrum problem. By elementary computation, the ordered 1-median values at $v^{*}$ and $v_{0}$ are $f_{\lambda}\left(v^{*}\right)=(4 n+1) B$ and $f_{\lambda}\left(v_{0}\right)=4 n B$, respectively. Moreover, either $v_{0}$ or $v^{*}$ is an anti- $(3 n+2)$-centrum of $T$ as other nodes are leaves. In what follows we prove that the answer to $(I)$ is 'yes' if and only if the answer to (InvAntiT) is 'yes'.

If the answer to $(I)$ is 'yes', there exists a subset $S^{\prime} \subset S$ such that $\sum_{a_{i} \in S^{\prime}} a_{i}=B$. We increase the length of $\left(v_{0}, v_{i}\right)$ by $2 a_{i}$ for $a_{i} \in S^{\prime}$ and get

$$
f_{\lambda}\left(v_{0}\right)=f_{\lambda}\left(v^{*}\right)=(4 n+2) B
$$

Furthermore, the modifying cost is $\sum_{a_{i} \in S^{\prime}} 2 a_{i}=2 B$, i.e., the answer to (InvAntiT) is 'yes'.

Conversely, suppose that the answer to $($ InvAntiT) is 'yes'. Then there exists a modification of edge lengths $\left(v_{0}, v_{i}\right)$ for $i=1, \ldots, n$ such that $\widetilde{f}_{\lambda}\left(v^{*}\right) \leq \widetilde{f}_{\lambda}\left(v_{0}\right)$ and the 
cost is at most $2 B$. If $\left(v_{0}, v_{i}\right)$ for some $i \in\{1, \ldots, n\}$ is increased by an amount $p_{i} \leq a_{i}$, the modification does not contribute to the improvement of the gap $f_{\lambda}\left(v_{0}\right)-f_{\lambda}\left(v^{*}\right)$. Therefore, if we increase $\left(v_{0}, v_{i}\right)$ by $p_{i}$, we can always assume that $p_{i}>a_{i}$. Let $I$ be the set of corresponding indices of the modified edges. We now prove that there exists at most one index $i_{0} \in I$ such that $\left(v_{0}, v_{i}\right)$ is increased by $2 a_{i}$ for all $i \in I \backslash\left\{i_{0}\right\}$. Indeed, assume that there exists $i \in I \backslash\left\{i_{0}\right\}$ such that $\left(v_{0}, v_{i}\right)$ is increased by an amount $p_{i}<2 a_{i}$. Let $\Delta=2 a_{i}-p_{i}$, we consider the following cases:

- If $\Delta \geq p_{i_{0}}-a_{i_{0}}$, we can set $p_{i}:=p_{i}+\left(p_{i_{0}}-a_{i_{0}}\right)$ and the edge length $\left(v_{0}, v_{i_{0}}\right)$ is fixed, i.e., its length is $B-a_{i_{0}}$. The gap $f_{\lambda}\left(v_{0}\right)-f_{\lambda}\left(v^{*}\right)$ is decreased by the same with a better cost. Therefore, we can iterate this procedure until this case does not occur.

- If $\Delta<p_{i_{0}}-a_{i_{0}}$, we assign $p_{i}:=2 a_{i}$ and $p_{i_{0}}:=p_{i_{0}}-\Delta>a_{i_{0}}$. Then the gap $f_{\lambda}\left(v_{0}\right)-f_{\lambda}\left(v^{*}\right)$ is reduced by the same and the cost does not change.

By the previous claim, we assume that $\left(v_{0}, v_{i_{0}}\right)$ is increased by $2 a_{i_{0}}-\delta$ with $\delta<a_{i_{0}}$ and $\left(v_{0}, v_{i}\right)$ is increased by $2 a_{i}$ for all $i \in I \backslash\left\{i_{0}\right\}$. The total cost is $\sum_{i \in I} 2 a_{i}-\delta \leq 2 B$ and the gap $f_{\lambda}\left(v^{*}\right)-f_{\lambda}\left(v_{0}\right) \leq 0$. Based on the computation of the gap, we obtain $\sum_{i \in I} a_{i} \leq B+\delta / 2$ and $\sum_{i \in I} a_{i} \geq B+\delta$. Thus, it yields $\delta=0$ and $\sum_{i \in I} a_{i}=B$. The result follows.

4. The inverse anti- $k$-centrum problem on unweighted cycles

\subsection{Property of an anti- $k$-centrum vertex in unweighted cycles}

We investigate in this section the inverse anti- $k$-centrum problem on an unweighted cycle, i.e., all node weights are equal to 1 . First, we explore some special properties of the corresponding anti- $k$-centrum problem. Given an unweighted cycle $C=(V, E)$ with clockwise numbered vertices, the vertex set is $V:=\left\{v_{0}, v_{1}, \ldots, v_{M-1}\right\}(M \geq 3)$, and the edge set is $E:=\left\{e_{1}, e_{2}, \ldots, e_{M}\right\}$, where $e_{i}:=\left(v_{i-1}, v_{i}\right)$ for $i=1, \ldots, M-1$ and supplemented by $e_{M}:=\left(v_{M-1}, v_{0}\right)$. For simplicity, if an index of a vertex or an edge exceeds $M-1$, then it is equivalent to its modulo of $M$.

First, the anti- $k$-centrum function $f_{\lambda}(\rho)$ for $\rho \in A(C)$ is concave in each edge of the cycle, see 25]. Therefore, we can directly derive the result.

Proposition 4.1. One of the vertices of $C$ is an anti-k-centrum of the cycle.

Proposition 4.1 allows us to evaluate the objective values only at the vertices of $C$ in order to get an optimal solution. Thus, a vertex $v^{*}$ is an anti-k-centrum of $C$ if and only if $f_{\lambda}\left(v^{*}\right) \leq f_{\lambda}(v)$ for all $v \in V$.

We will show in what follows that the anti- $k$-centrum objective function at any vertex on $C$ is related to the 1-median objective function of that vertex on paths and 1-medians 
of subpaths with $k$ vertices in $C$ play an important role in finding an anti- $k$-centrum of the cycle. Therefore, we decompose the cycle $C$ into the subpaths, each subpath contains exactly $k$ vertices, $k \leq M$. Then we aim to explore a special property of a 1-median on each of these subpaths to establish an optimality criterion for an anti- $k$-centrum of an unweighted cycle.

Denote by $\mathcal{P}^{k}$ the set of all subpaths of $C$ with exactly $k$ vertices, one observes that $\left|\mathcal{P}^{k}\right|=M$. Let $P:=(V(P), E(P)) \in \mathcal{P}^{k}$, we now define the objective value of a vertex $v$ on $P$. Notice that there are two different types of distances from a vertex $v^{\prime} \in P$ to $v$. First, if we consider the distance from $v^{\prime}$ to $v$ in the cycle $C$, we get the usual distance denoted by $d\left(v, v^{\prime}\right)$. On the other hand, if we consider the distance from $v^{\prime}$ to $v$ in the path $P$, we get another type of distance denoted by $d^{P}\left(v, v^{\prime}\right)$. It is obvious that $d\left(v, v^{\prime}\right) \leq d^{P}\left(v, v^{\prime}\right)$. We consider the objective value of $v$ on the path $P$ as follows.

Definition 4.2. The objective value of a vertex $v \in V(P)$ on the path $P$ is defined as

$$
\Omega^{P}(v):=\sum_{v^{\prime} \in P} d^{P}\left(v, v^{\prime}\right)
$$

From now on, we present a path with $k$ vertices as $P:=\left(v_{i_{1}}, v_{i_{2}}, \ldots, v_{i_{k}}\right)$ in that order. It means that for two arbitrary adjacent vertices $v_{i_{j}}, v_{i_{j+1}}$ in $P$, we identify an edge $\left(v_{i_{j}}, v_{i_{j+1}}\right)$.

Denote by $\mathcal{P}(v)$ the set of all paths in $\mathcal{P}^{k}$ that contains the vertex $v$. Then the relation of the objective value at $v$ in $C$ and the objective values of $v$ on the paths in $\mathcal{P}(v)$ is expressed in the following lemma.

Lemma 4.3. The anti-k-centrum objective value at a vertex $v$ on the cycle $C$ is identified by

$$
F_{\lambda}(v)=\min _{P \in \mathcal{P}(v)} \Omega^{P}(v)
$$

Lemma 4.3 is obviously correct as the vertices whose distances contributed to $F_{\lambda}(v)$ is connected. By Lemma 4.3, for each $v \in C$ there exists a path $P_{v} \in \mathcal{P}(v)$ such that $F_{\lambda}(v)=\Omega^{P_{v}}(v)$. The path $P_{v}$ is called the induced path of $v$ in $C$. A special property of induced paths of an anti- $k$-centrum in $C$ is studied in the following lemma.

Lemma 4.4. Given a cycle $C$ and $v^{*}$ is an anti-k-centrum of $C$. Then $v^{*}$ is a 1-median of its induced path, i.e.,

$$
\sum_{v^{\prime} \in P_{v^{*}}} d_{\ell}^{P_{v^{*}}}\left(v^{\prime}, v^{*}\right) \leq \sum_{v^{\prime} \in P_{v^{*}}} d_{\ell}^{P_{v^{*}}}\left(v^{\prime}, v\right)
$$

for all vertex $v$ in an induced path $P_{v^{*}}$ of $v^{*}$. 
Proof. Assume that $v^{*}$ is an anti- $k$-centrum of $C$ but it is not a 1-median of its induced path $P_{v^{*}}$. Then, there exists a vertex $v_{i_{0}} \in P_{v^{*}}$ such that $F_{\lambda}\left(v^{*}\right)=\Omega^{P_{v^{*}}}\left(v^{*}\right)>\Omega^{P_{v^{*}}}\left(v_{i_{0}}\right)$. However, it implies that $\Omega^{P_{v^{*}}}\left(v_{i_{0}}\right) \geq F_{\lambda}\left(v_{i_{0}}\right)$ by Lemma 4.3 . Thus, $F_{\lambda}\left(v^{*}\right)>F_{\lambda}\left(v_{i_{0}}\right)$ or the optimality of $v^{*}$ in $C$ is contradicted. The result follows.

Lemma 4.4 states that an anti- $k$-centrum of $C$ is also a 1-median of its induced path. Thus, for each $v \in C$ we concentrate on the path such that $v$ is its 1-median. If $P$ is a path in $C$ such that $v$ is a 1-median of $P$, then we define $P$ as the candidate path of $v$ and the corresponding value $\Omega^{P}(v)$ is called candidate objective value of $v$. Due to Lemma 4.4 . we can observe that the necessary condition for a vertex $v$ to be an anti- $k$-centrum of $C$ is that it is a 1-median of its induced path. Therefore, we are going to investigate the sufficient condition for a vertex $v^{*}$ to be the anti- $k$-centrum of the cycle $C$ as follows.

Theorem 4.5 (Optimality criterion). Given an unweighted cycle $C$ and a vertex $v^{*} \in C$. Then $v^{*}$ is an anti-k-centrum of $C$ if and only if there exists a candidate path $P$ of $v$ such that $\Omega^{P}\left(v^{*}\right) \leq \Omega^{P^{\prime}}\left(v^{\prime}\right)$ for all candidate paths $P^{\prime}$ of $v^{\prime} \neq v^{*}, \forall v^{\prime} \in V$.

Proof. First of all, if $v^{*}$ is an anti- $k$-centrum of $C$, there exists a candidate path $P_{v^{*}} \in$ $\mathcal{P}\left(v^{*}\right)$ of $v^{*}$ such that $F_{\lambda}\left(v^{*}\right)=\Omega^{P_{v^{*}}}\left(v^{*}\right)$ by Lemma 4.4. Since $F_{\lambda}\left(v^{\prime}\right)=\min _{P^{\prime} \in \mathcal{P}\left(v^{\prime}\right)} \Omega^{P^{\prime}}\left(v^{\prime}\right)$ and $F_{\lambda}\left(v^{*}\right) \leq F_{\lambda}\left(v^{\prime}\right)$ for all $v^{\prime} \in C \backslash\left\{v^{*}\right\}$, we get $\Omega^{P_{v^{*}}}\left(v^{*}\right) \leq \Omega^{P^{\prime}}\left(v^{\prime}\right)$ for all candidate paths $P^{\prime}$ of $v^{\prime} \neq v^{*}$.

Conversely, assume that there exists a candidate path $P_{v^{*}}$ of $v^{*}$ such that $\Omega^{P_{v^{*}}}\left(v^{*}\right) \leq$ $\Omega^{P^{\prime}}\left(v^{\prime}\right)$ for all candidate paths $P^{\prime}$ of $v^{\prime} \neq v^{*}$. First, we claim that $F_{\lambda}\left(v^{*}\right)=\Omega^{P^{*}}\left(v^{*}\right)$ for some candidate path $P^{*}$ of $v^{*}$. Indeed, assume that $F_{\lambda}\left(v^{*}\right)=\Omega^{P^{\prime}}\left(v^{*}\right)$ and $P^{\prime}$ is not a candidate path of $v^{*}$, then there exists a 1-median $v^{\prime}$ of $P^{\prime}$ such that $\Omega^{P^{\prime}}\left(v^{*}\right)>\Omega^{P^{\prime}}\left(v^{\prime}\right)$. We trivially get $\Omega^{P}\left(v^{*}\right) \geq \Omega^{P^{\prime}}\left(v^{*}\right)>\Omega^{P^{\prime}}\left(v^{\prime}\right)$ for all candidate paths $P$ of $v^{*}$. It contradicts to the optimality of $v^{*}$. Assume by contradiction that $v^{*}$ is not an anti-k-centrum of $C$, then there exists an anti- $k$-centrum $v^{\prime}$ of $C$ such that $F_{\lambda}\left(v^{\prime}\right)<F_{\lambda}\left(v^{*}\right)$. By Lemma 4.4 . there exists a candidate path $P^{\prime}$ of $v^{\prime}$ such that $F_{\lambda}\left(v^{\prime}\right)=\Omega^{P^{\prime}}\left(v^{\prime}\right)$. However, this contradicts to the assumption as

$$
\Omega^{P^{*}}\left(v^{*}\right) \leq \Omega^{P_{v^{*}}}\left(v^{*}\right) \leq \Omega^{P^{\prime}}\left(v^{\prime}\right) .
$$

Therefore, we can finally conclude that $v^{*}$ is an anti- $k$-centrum of $C$.

Theorem 4.5 helps us to evaluate the optimal objective value of an anti- $k$-centrum problem on an unweighted cycle by computing the objective value of each vertex $v$ on its candidate path(s) and get the minimum one. The next task is to identify the candidate path of a given vertex $v$. Remind that we number the vertices of $C$ in clockwise direction. For a given vertex $v_{i} \in C$, we can find its candidate path(s) as in follows. 
Proposition 4.6. Given an unweighted cycle $C=(V, E)$ with clockwise-numbered vertices $v_{0}, \ldots, v_{M-1}$. For a given vertex $v_{i}$, we get the two following cases:

(1) If $k=2 m+1$, there is exactly one candidate path of $v_{i}$, say

$$
P_{0}^{i}=\left(v_{i-m}, \ldots, v_{i}, \ldots, v_{i+m}\right) \text {. }
$$

(2) If $k=2 m$, there are exactly two candidate paths of $v_{i}$, say

$$
P_{1}^{i}=\left(v_{i-m}, \ldots, v_{i}, \ldots, v_{i+m-1}\right) \quad \text { and } \quad P_{2}^{i}=\left(v_{i-m+1}, \ldots, v_{i}, \ldots, v_{i+m}\right) .
$$

Proof. The proof is omitted since the result can be directly derived from the optimality criterion for a prespecified vertex to be a 1-median of a tree, see Goldman 17 and Hua 19 .

From Proposition 4.6, we observe that there exist one or two candidate paths of a vertex $v_{i} \in C$ depending on the odd or even integer $k$. For simplicity, we denote by $\Omega\left(v_{i}\right)$ the objective value of $v_{i}$ on its candidate path $P_{0}^{i}$ if $k$ is odd. In case $k$ is even, denote by $\Omega_{1}\left(v_{i}\right)$ and $\Omega_{2}\left(v_{i}\right)$ the objective values of $v_{i}$ on its two candidate paths $P_{1}^{i}$ and $P_{2}^{i}$, respectively. Here, the candidate $\operatorname{path}(\mathrm{s})$ of $v_{i}$ is implicitly involved in the functions $\Omega$, $\Omega_{1}$ and $\Omega_{2}$. In summary, we can mathematically write

$$
\Omega\left(v_{i}\right)=\Omega^{P_{0}^{i}}\left(v_{i}\right) \text { for } i=0, \ldots, M-1 \text { if } k \text { is odd }
$$

and

$$
\Omega_{j}\left(v_{i}\right)=\Omega^{P_{j}^{i}}\left(v_{i}\right) \quad \text { for } i=0, \ldots, M-1 \text { and } j=1,2 \text { if } k \text { is even. }
$$

Combining Proposition 4.6 and Theorem 4.5 , we get

Corollary 4.7 (Optimality criterion, a reformulation of Theorem 4.5). Given an unweighted cycle $C$ and a prespecified vertex $v^{*}$. Then $v^{*}$ is an anti-k-centrum of $C$ if and only if the following condition holds

(1) $\Omega\left(v^{*}\right) \leq \Omega(v)$ for all $v \neq v^{*}$, if $k$ is odd.

(2) $\Omega_{1}\left(v^{*}\right) \leq \Omega_{j}(v)$ for all $v \neq v^{*}, j=1,2$ or $\Omega_{2}\left(v^{*}\right) \leq \Omega_{j}(v)$ for all $v \neq v^{*}, j=1,2$, if $k$ is even.

In case $k=2 m$ is an even integer, by elementary computation we can directly derive the relation of objective values of vertices with respect to their candidate paths as in the lemma below.

Lemma 4.8. Given an even integer $k$ and an unweighted cycle $C$, it holds that

$$
\Omega_{1}\left(v_{i+1}\right)=\Omega_{2}\left(v_{i}\right) \quad \text { for } i=0, \ldots, M-1 .
$$


From Lemma 4.8, we concentrate on the candidate optimal objective values of vertices with respect to function $\Omega_{1}$ (or $\Omega_{2}$ ) in order to find an anti-k-centrum of $C$. In more words, we calculate the value $\Omega_{1}\left(v_{i}\right)$ for $i=0, \ldots, M-1$ and select the minimum one. The attained value is the optimal objective value and the vertex with respect to that value is an anti- $k$-centrum of $C$. In conclusion, we get the result.

Corollary 4.9. An anti-k-centrum vertex of an unweighted cycle can be found in linear time.

\subsection{Solution approach and some special cases}

\subsubsection{General solution approach}

In this section we consider a general solution method for the inverse anti- $k$-centrum problem on an unweighted cycle $C=(V, E)$. Without loss of generality, suppose that the prespecified vertex $v^{*}$ coincides with $v_{0}$. Our goal is to modify the edge lengths of the cycle at minimum cost such that $v_{0}$ becomes an anti- $k$-centrum of $C$. We now use the abbreviation $\widetilde{\Omega}(v)$ or $\widetilde{\Omega}_{1}(v), \widetilde{\Omega}_{2}(v)$ to represent the objective value of $v$ on its candidate path(s) with respect to the new edge lengths $\widetilde{\ell}_{e}=\ell_{e}+p_{e}-q_{e}$ for $e \in E$.

Recall the optimality criterion in Corollary 4.7, the vertex $v_{0}$ is an anti- $k$-centrum of $C$ if and only if one of the objective value(s) at $v_{0}$ with respect to its candidate path(s) (possibly two paths) is the smallest in comparison to those of the other vertices. Moreover, by Lemma 4.8 we just compare $\Omega_{1}\left(v_{0}\right)$ and $\Omega_{2}\left(v_{0}\right)$ with $\Omega_{1}\left(v_{i}\right)$ for $i=1, \ldots, M-1$ in order to decide if $v_{0}$ is an anti- $k$-centrum of $C$ or not. For simplicity, denote by $p_{j}$ and $q_{j}$ the amount of augmentation and reduction of length of edge $e_{j}$ for $j=1, \ldots, M$, respectively. Then we consider two cases:

1. If $k$ is odd, i.e., $k=2 m+1 \leq M$, then we trivially get the formulation of $(\operatorname{Inv} C)$ :

$$
\begin{array}{ll}
\min & \sum_{j=1}^{M}\left(c_{j}^{+} p_{j}+c_{j}^{-} q_{j}\right) \\
\text { s.t. } & \widetilde{\Omega}\left(v_{0}\right) \leq \widetilde{\Omega}\left(v_{i}\right), \forall i=1, \ldots, M-1, \quad 0 \leq p_{j} \leq \bar{p}_{j}, \forall j=1, \ldots, M, \\
& 0 \leq q_{j} \leq \bar{q}_{j}, \forall j=1, \ldots, M .
\end{array}
$$

2. If $k$ is even, i.e., $k=2 m \leq M$, then we can formulate (InvC) through two subproblems as follows:

$$
\begin{array}{ll}
\min & \sum_{j=1}^{M}\left(c_{j}^{+} p_{j}+c_{j}^{-} q_{j}\right) \\
\text { s.t. } & \widetilde{\Omega}_{1}\left(v_{0}\right) \leq \widetilde{\Omega}_{1}\left(v_{i}\right), \forall i=2, \ldots, M-1, \quad 0 \leq p_{j} \leq \bar{p}_{j}, \forall j=1, \ldots, M, \\
& 0 \leq q_{j} \leq \bar{q}_{j}, \forall j=1, \ldots, M
\end{array}
$$


and

$$
\begin{array}{ll}
\min & \sum_{j=1}^{M}\left(c_{j}^{+} p_{j}+c_{j}^{-} q_{j}\right) \\
\text { s.t. } & \widetilde{\Omega}_{2}\left(v_{0}\right) \leq \widetilde{\Omega}_{1}\left(v_{i}\right), \forall i=2, \ldots, M-1, \quad 0 \leq p_{j} \leq \bar{p}_{j}, \forall j=1, \ldots, M, \\
& 0 \leq q_{j} \leq \bar{q}_{j}, \forall j=1, \ldots, M .
\end{array}
$$

Note that in 4.2 we do not add the constraint $\widetilde{\Omega}_{1}\left(v_{0}\right) \leq \widetilde{\Omega}_{1}\left(v_{1}\right)$ since $\widetilde{\Omega}_{1}\left(v_{1}\right)=\widetilde{\Omega}_{2}\left(v_{0}\right)$. Therefore, the constraint $\widetilde{\Omega}_{1}\left(v_{0}\right) \leq \widetilde{\Omega}_{1}\left(v_{1}\right)$ does not effect on the optimality of $v_{0}$. By the same argument, in 4.3 we skip the constraint $\widetilde{\Omega}_{2}\left(v_{0}\right) \leq \widetilde{\Omega}_{1}\left(v_{1}\right)$. To get the optimal objective value of the inverse anti- $k$-centrum problem, we compare the optimal objective values of 4.2 and 4.3 and take the smaller one.

The functions $\widetilde{\Omega}(\cdot), \widetilde{\Omega}_{1}(\cdot)$ and $\widetilde{\Omega}_{2}(\cdot)$ are linear in variable edge lengths. Moreover, the new edge lengths can be presented linearly in the variables $p_{j}$ and $q_{j}$ for $j=1, \ldots, M$. Therefore, the formulations (4.1), (4.2) and (4.3) are indeed linear programs.

Theorem 4.10. The inverse anti-k-centrum problem on an unweighted cycle can be translated to one or two linear programming problems depending on odd or even $k$.

Next we aim to translate 4.1), (4.2), (4.3) to a more explicit formulation such that they can be better implemented. First we introduce an operator as below.

Definition 4.11. A pushing operator $\pi_{i}: \mathbb{R}^{M} \rightarrow \mathbb{R}^{M}$ for $i \in \mathbb{N}$ is a function defined as follows. Given a vector $x=\left(x_{1}, x_{2}, \ldots, x_{M}\right) \in \mathbb{R}^{M}$ then

$$
\pi_{i}(x)=\left(x_{M-i+1}, x_{M-i+2}, \ldots, x_{M}, x_{1}, x_{2}, \ldots, x_{M-i}\right)
$$

The pushing operator $\pi_{i}$ pushes the index of each component of vector $x$ by $i$ position on its right-hand side. If the index of a component in $\pi_{i}(x)$ is larger than $M$, the corresponding new index is its modulo of $M$. Thus, we can note that $\pi_{M}(x)=x$, $\pi_{M+1}(x)=\pi_{1}(x)$ and so on. Additionally, we define $\pi_{0}(x)=x$. For example, given a vector $x=(1,2,3,4,5)$ on $\mathbb{R}^{4}$, then $\pi_{1}(x)=(5,1,2,3,4), \pi_{2}(x)=(4,5,1,2,3)$ and so on.

Now we consider the basis vector(s) which contain the multiplication of edges in the candidate path of $v_{0}$. We classify these two cases:

1. If $k=2 m+1$ is an odd integer, we denote by

$$
a=(m, m-1, \ldots, 1,0, \ldots, 0,1, \ldots, m-1, m) \in \mathbb{R}^{M} .
$$

2. If $k=2 m$ is an even integer, we denote by

$$
a^{1}=(m-1, m-2, \ldots, 1,0, \ldots, 0,1, \ldots, m-1, m) \in \mathbb{R}^{M}
$$


and

$$
a^{2}=(m, m-1, \ldots, 1,0, \ldots, 0,1, \ldots, m-2, m-1) \in \mathbb{R}^{M}
$$

Additionally, the corresponding vector of edge lengths in $C$ before and after the modification is denoted by $\ell=\left(\ell_{e_{1}}, \ell_{e_{2}}, \ldots, \ell_{e_{M}}\right)$ and $\widetilde{\ell}=\left(\widetilde{\ell}_{e_{1}}, \widetilde{\ell}_{e_{2}}, \ldots, \widetilde{\ell}_{e_{M}}\right)$, respectively. Then we can represent the objective value of $v_{i}$ in its candidate path(s) in the following proposition.

Proposition 4.12. If $k=2 m+1$ is an odd integer, the candidate objective value of $v_{i}$ is

$$
\Omega\left(v_{i}\right)=\pi_{i}(a)^{T} \ell \quad \text { for } i=0,1,2, \ldots, M-1 .
$$

In case $k=2 m$ is an even integer, the candidate objective values of $v_{i}$ are

$$
\Omega_{1}\left(v_{i}\right)=\pi_{i}\left(a^{1}\right)^{T} \ell \quad \text { and } \quad \Omega_{2}\left(v_{i}\right)=\pi_{i}\left(a^{2}\right)^{T} \ell \quad \text { for } i=0,1,2, \ldots, M-1 .
$$

Now we can present the candidate value(s) of each vertex as a linear function of edge length modifications. Denote by $p=\left(p_{j}\right)_{j=1, \ldots, M}$ and $q=\left(q_{j}\right)_{j=1, \ldots, M}$. Let us consider two following cases:

1. If $k=2 m+1$ is an odd integer, then

$$
\widetilde{\Omega}\left(v_{i}\right)=\Omega\left(v_{i}\right)+\pi_{i}(a)^{T}(p-q) \quad \text { for } i=0, \ldots, M-1 .
$$

We can transform 4.1 into the following linear programming problem:

$$
\begin{array}{ll}
\min & \sum_{j=1}^{M}\left(c_{j}^{+} p_{j}+c_{j}^{-} q_{j}\right) \\
\text { s.t. } & \left(\pi_{i}(a)-a\right)^{T}(p-q) \geq b_{i}, \forall i=1, \ldots, M-1, \\
& 0 \leq p_{j} \leq \bar{p}_{j}, \forall j=1, \ldots, M, \quad 0 \leq q_{j} \leq \bar{q}_{j}, \forall j=1, \ldots, M,
\end{array}
$$

where $b_{i}=\Omega\left(v_{0}\right)-\Omega\left(v_{i}\right)$ for $i=1, \ldots, M-1$.

2. If $k=2 m$ is an even integer, then by the similar computation in the first case we can also transform 4.2 and 4.3 into the two linear programming problems:

$$
\begin{array}{ll}
\min & \sum_{j=1}^{M}\left(c_{j}^{+} p_{j}+c_{j}^{-} q_{j}\right) \\
\text { s.t. } & \left(\pi_{i}\left(a^{1}\right)-a^{1}\right)^{T}(p-q) \geq b_{i}^{1}, \forall i=2, \ldots, M-1, \\
& 0 \leq p_{j} \leq \bar{p}_{j}, \forall j=1, \ldots, M, \quad 0 \leq q_{j} \leq \bar{q}_{j}, \forall j=1, \ldots, M
\end{array}
$$


and

$$
\begin{array}{ll}
\min & \sum_{j=1}^{M}\left(c_{j}^{+} p_{j}+c_{j}^{-} q_{j}\right) \\
\text { s.t. } & \left(\pi_{i}\left(a^{2}\right)-a^{1}\right)^{T}(p-q) \geq b_{i}^{2}, \forall i=2, \ldots, M-1, \\
& 0 \leq p_{j} \leq \bar{p}_{j}, \forall j=1, \ldots, M, \quad 0 \leq q_{j} \leq \bar{q}_{j}, \forall j=1, \ldots, M,
\end{array}
$$

where $b_{i}^{1}=\Omega_{1}\left(v_{0}\right)-\Omega_{1}\left(v_{i}\right), b_{i}^{2}=\Omega_{2}\left(v_{0}\right)-\Omega_{1}\left(v_{i}\right)$ for $i=2, \ldots, M-1$.

\subsubsection{Some special cases of $(\operatorname{Inv} C)$}

We investigate in this section some special cases of the inverse anti-k-centrum problem on an unweighted cycle $C$ with efficient solution methods. If $k=1$, every vertex of the cycle $C$ is an anti-1-centrum as their corresponding objective values all equal 0 . Thus, we explore some special cases with $k \geq 2$.

- The inverse anti-2-centrum problem on cycles.

For $k=2$, the vector of multipliers is

$$
\lambda=(1,1,0, \ldots, 0) \in \mathbb{R}^{M}
$$

First of all, we observe that the smallest edge length plays an important role in identifying an anti-2-centrum of the underlying cycle.

Theorem 4.13 (Optimality criterion). A vertex $v$ is an anti-2-centrum of $C$ if an only if there exists an incident edge of $v$ that has the smallest length in the cycle.

From Theorem 4.13, we modify the edge lengths in $C$ in minimum cost such that $e_{1}$ or $e_{M}$ becomes the smallest one. Assume that we want $e_{1}$ to become the smallest edge among $e_{1}, e_{2}, \ldots, e_{M-1}$, then we have the following lemma.

Lemma 4.14. In the optimal modification such that $e_{1}$ becomes the smallest edge, it is sufficient to decrease the length of $e_{1}$ and increase the length of edges $e_{2}, e_{3}, \ldots, e_{M-1}$.

We denote by $c$ the cost to decrease one unit edge length and $\ell$ the current length of $e_{1}$. Moreover, let $c_{j}$ be the cost to increase one unit edge length and $\ell_{j}$ be the length of $e_{j}$ for $j=2, \ldots, M-1$. Let $\xi$ be the modifying length of $e_{1}$. Denote by $\ell_{\min }=\min _{j=2, \ldots, M-1} \ell_{j}$, in order to perturbe $e_{1}$ into the smallest edge length we decrease the length of $e_{1}$ to $\ell_{\min }$ in the worst case. Thus, we get the lower bound of $\xi$ :

$$
\max \left\{\ell_{\min }, \ell-\bar{q}_{1}\right\} \leq \xi
$$


Let $\ell_{\max }=\min _{j=2, \ldots, M-1}\left(\ell_{j}+\bar{p}_{j}\right)$, then in the worst case we have to decrease the length of $e_{1}$ to $\ell_{\max }$ and increase the lengths of $e_{j}$ for $\ell_{j}<\ell_{\max }$ to $\ell_{\max }$ in order to modify the length of $e_{1}$ to the smallest edge length. Thus, we get

$$
\xi \leq \min \left\{\ell_{\max }, \ell\right\}
$$

We abbreviate by $\underline{\xi}=\max \left\{\ell_{\min }, \ell-\bar{q}_{1}\right\}$ and $\bar{\xi}=\min \left\{\ell_{\max }, \ell\right\}$. After the preprocessing step, we get the following problem

$$
\text { minimize } \quad C(\xi):=c(\ell-\xi)+\sum_{e_{j}: \ell_{j}<\xi} c_{j}\left(\xi-\ell_{j}\right) \quad \text { for } \underline{\xi} \leq \xi \leq \bar{\xi}
$$

Problem 4.5 states that we should decrease the length of edge $e_{1}$ to $\xi$, the cost is $c(\ell-\xi)$. On the other hand, other edges with the length being strictly less than $\xi$ should be increased to $\xi$, the cost is $c_{j}\left(\xi-\ell_{j}\right)$ for $\ell_{j}<\xi$. Since the function $C(\xi)$ is piecewise linear and convex, the minimum value of $C(\xi)$ can be found in linear time by the binary seach algorithm proposed in Alizadeh and Burkard [4. Similarly, we solve the same problem in which $e_{M}$ becomes the smallest edge length and get another optimal objective value. Finally, we compare these two values and choose the best one. In conclusion, we get the following result.

Theorem 4.15. The inverse anti-2-centrum problem on unweighted cycles is solvable in linear time.

- The inverse anti-3-centrum problem on cycles.

We now consider the case $k=3$. Then the corresponding basis vector is $a=$ $(1,0, \ldots, 0,1)$ and the matrix of coefficients $A=\left(\begin{array}{c}\pi_{1}(a)-a \\ \pi_{2}(a)-a \\ \ldots \ldots \ldots \\ \pi_{M-1}(a)-a\end{array}\right)$ with respect to the constraints of (4.4) is represented as

$$
A=\left(\begin{array}{ccccccc}
0 & 1 & 0 & \cdots & 0 & 0 & -1 \\
-1 & 1 & 1 & \cdots & 0 & 0 & -1 \\
-1 & 0 & 1 & \cdots & 0 & 0 & -1 \\
\cdots & \cdots & \cdots & \cdots & \cdots & \cdots & \cdots \\
-1 & 0 & 0 & \cdots & 1 & 0 & -1 \\
-1 & 0 & 0 & \cdots & 1 & 1 & -1 \\
-1 & 0 & 0 & \cdots & 0 & 1 & 0
\end{array}\right) \in \mathbb{R}^{(M-1) \times M}
$$


Denote by $p$ and $q$ the vector of augmentation and reduction of edge lengths, respectively. Moreover, let $\bar{p}$ and $\bar{q}$ be the vector of upper bounds of augmentation and reduction of edge lengths. The inverse anti-3-centrum problem on $C$ is then formulated as

$$
\min \sum_{j=1}^{M}\left(c_{j}^{+} p_{j}+c_{j}^{-} q_{j}\right) \quad \text { s.t. } \quad A(p-q) \geq b, 0 \leq p \leq \bar{p}, 0 \leq q \leq \bar{q} .
$$

Here, on the right-hand side of $(4.6), b=\left(\begin{array}{c}\Omega\left(v_{0}\right)-\Omega\left(v_{1}\right) \\ \Omega\left(v_{0}\right)-\Omega\left(v_{2}\right) \\ \ldots \ldots \ldots \ldots \ldots \\ \Omega\left(v_{0}\right)-\Omega\left(v_{M-1}\right)\end{array}\right)$.

From the special structure of matrix $A$, we can presolve 4.6 by fixing some variables.

Proposition 4.16. In the inverse anti-3-centrum problem formulated as (4.6) we can assign

$$
p_{1}=p_{M}=0 \quad \text { and } \quad q_{2}=q_{3}=\cdots=q_{M-1}=0,
$$

without changing the problem.

Thus, we can replace $p-q$ in 4.6 by $x \in \mathbb{R}^{M}$ where $\bar{x}_{1}=\bar{q}_{1}, \bar{x}_{M}=\bar{q}_{M}$ and $\bar{x}_{j}=\bar{p}_{j}$ for $j=2,3, \ldots, M-1$. Let $c_{j}=c_{j}^{-}$if $j=1$ or $j=M$, and $c_{j}=c_{j}^{+}$otherwise. Moreover, let us denote by

$$
A^{\prime}=\left(\begin{array}{ccccccc}
0 & 1 & 0 & \cdots & 0 & 0 & 1 \\
1 & 1 & 1 & \cdots & 0 & 0 & 1 \\
1 & 0 & 1 & \cdots & 0 & 0 & 1 \\
\cdots & \ldots & \ldots & \cdots & \cdots & \ldots & \ldots \\
1 & 0 & 0 & \cdots & 1 & 0 & 1 \\
1 & 0 & 0 & \cdots & 1 & 1 & 1 \\
1 & 0 & 0 & \cdots & 0 & 1 & 0
\end{array}\right) .
$$

Additionally, let $\bar{x}$ be the vector of upper bounds of components of $x$ then we can reformulate 4.6 as

$$
\min \sum_{j=1}^{M} c_{j} x_{j} \quad \text { s.t. } \quad A^{\prime} x \geq b, 0 \leq x \leq \bar{x} .
$$

Matrix $A^{\prime}$ is a consecutive 1's matrix. Thus, we can translate 4.7) into an equivalent minimum cost flow problem, it is thus solvable efficiently; see Ahuja et al. [1].

Theorem 4.17. The inverse anti-3-centrum problem on a cycle can be reduced to a minimum cost flow problem in linear time. 
Theorem 4.17 states that we can use the methods to solve the minimum cost flow problems to deal with the inverse anti-3-centrum problem. Now we explore the problem with uniform-cost coefficients.

Uniform-cost inverse anti-3-centrum problem on $C($ InvU3C):

Let the cost coefficients be all equal to 1, we now derive an efficient solution approach for $(\operatorname{Inv} U 3 C)$. By the special structure of matrix $A^{\prime}$ in (4.7), we first consider the distribution of variables $x_{1}$ and $x_{M}$. The following result is obvious.

Proposition 4.18. If $b_{1}-\bar{x}_{M}, b_{M-1}-\bar{x}_{1}$, and $b_{i}-\bar{x}_{1}-\bar{x}_{M}$ for $i=2, \ldots, M-2$ are nonpositive, then the optimal objective of $\left(\right.$ InvU3C) is less than or equal to $\bar{x}_{1}+\bar{x}_{M}$.

The conditions given in Proposition 4.18 can be checked in linear time by computing all corresponding differences. If these conditions hold, we can directly solve the problem by focusing on the distribution of two variables $x_{1}$ and $x_{M}$. Without loss of generality, we can assume that $b_{1}$ and $b_{M-1}$ are positive. Otherwise, we can replace $b_{1}$ or $b_{M-1}$ by zero. Set $x_{1}:=b_{M-1}$ and $x_{M}:=b_{1}$. The updated upper bound is $\bar{x}_{1}:=\bar{x}_{1}-b_{M-1}$ and $\bar{x}_{M}:=\bar{x}_{M}-b_{1}$ and set $b_{i}:=b_{i}-b_{1}-b_{M-1}$ for $i=2, \ldots, M-2$. If $b_{i} \leq 0$ for $i=2, \ldots, M-2$, the optimal cost is $b_{1}+b_{M-1}$. Otherwise, we set $b_{i_{0}}:=\max _{i=2, \ldots, M-2}\left\{b_{i}\right\}$ and distribute $x_{1}$ and $x_{M}$ such that $x_{1}+x_{M}=b_{i_{0}}$. The optimal objective value is $b_{i_{0}}+b_{1}+b_{M}$.

Next we consider the case where conditions in Proposition 4.18 do not hold. After setting $x_{1}:=\bar{x}_{1}$ and $x_{M}:=\bar{x}_{M}$ and updating $b_{1}:=b_{1}-\bar{x}_{M}, b_{M-1}:=b_{M-1}-\bar{x}_{1}$, and $b_{i}:=b_{i}-\bar{x}_{1}-\bar{x}_{M}$ for $i=2, \ldots, M-2$, we can rewrite (4.7) as follows:

$$
\min \sum_{j=2}^{M-2} x_{j} \quad \text { s.t. } \quad \widetilde{A} \widetilde{x} \geq b, 0 \leq x_{j} \leq \bar{x}_{j}, \quad \forall j=2 \ldots, M-1 .
$$

Here, the matrix $\widetilde{A}$ is

$$
\widetilde{A}=\left(\begin{array}{ccccc}
1 & 0 & \cdots & 0 & 0 \\
1 & 1 & \cdots & 0 & 0 \\
0 & 1 & \cdots & 0 & 0 \\
\cdots & \cdots & \cdots & \cdots & \cdots \\
0 & 0 & \cdots & 1 & 0 \\
0 & 0 & \cdots & 1 & 1 \\
0 & 0 & \cdots & 0 & 1
\end{array}\right) \in \mathbb{R}^{(M-1) \times(M-2)}
$$

and $\widetilde{x}=\left(x_{2}, x_{3}, \ldots, x_{M-1}\right)$. Let $x_{j+1}=y_{j}$ for $j=1, \ldots, M-2$ and $n=M-2$. Then we get the vector of new variables $\left(y_{1}, \ldots, y_{n}\right)$, with $\bar{y}_{j}=\bar{x}_{j+1}$ for $j=1, \ldots, n$. The optimal objective value of $(\operatorname{Inv} U 3 C)$ is equal to the optimal objective value of (4.8) adding $\bar{x}_{1}+\bar{x}_{M}$. 
Because of the special structure of 4.8, we can design Algorithm 4.1 to solve it efficiently. The main idea of Algorithm 4.1 is to go step by step downward of the constraints and find the minimum values of variables such that those constraints hold.

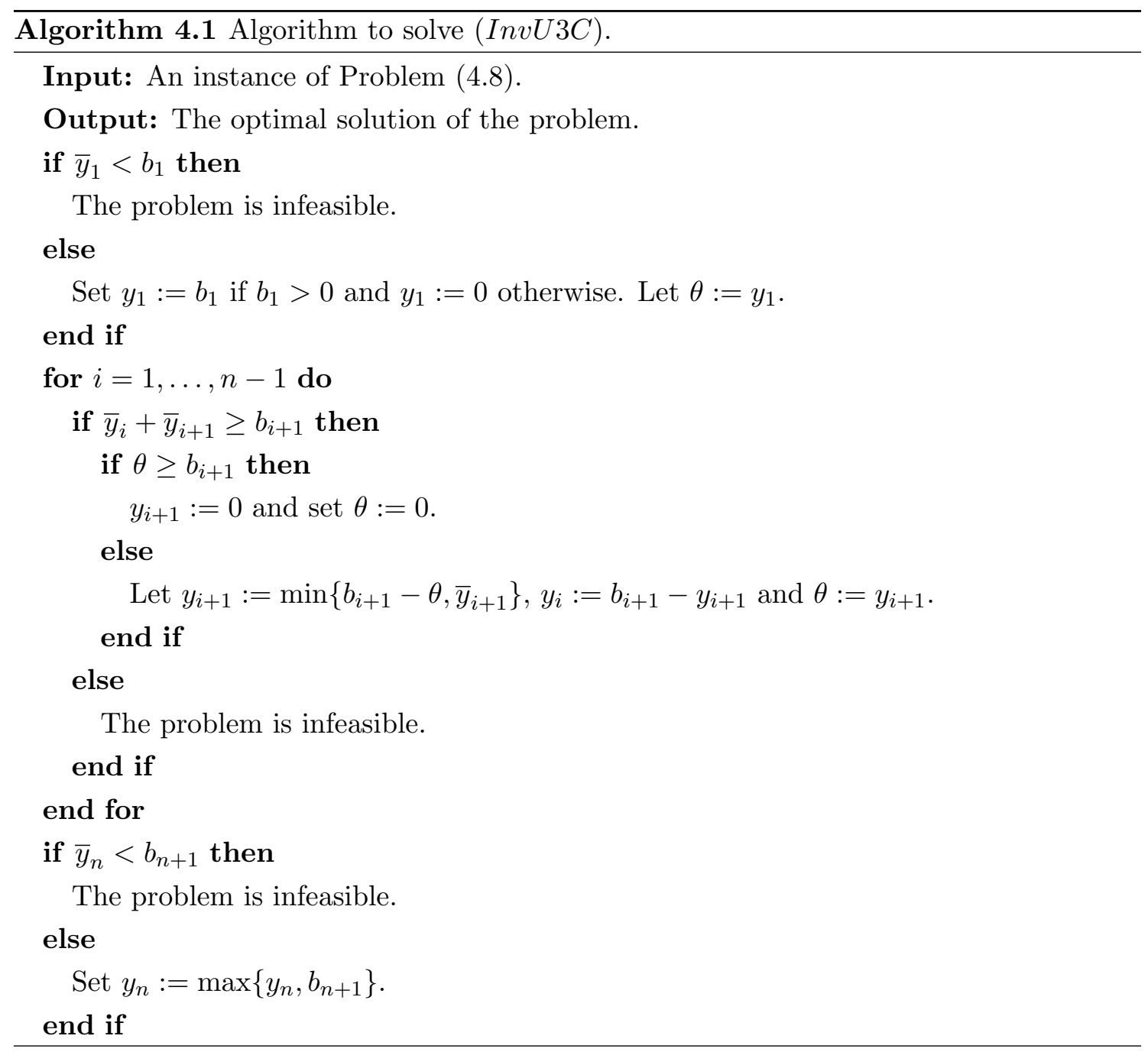

Algorithm 4.1 runs in linear time as we iterate each row of 4.8 once. The correctness of Algorithm 4.1 is shown as follows.

Proposition 4.19. Algorithm 4.1 finds an optimal solution of 4.8.

Proof. Without loss of generality, we can assume that the problem is feasible. To show that the obtained solution is optimal, we apply the induction on the number of variables.

If $n=1$, the corresponding problem is

$$
\min \quad y_{1} \quad \text { s.t. } \quad y_{1} \geq b_{1}, 0 \leq y_{1} \leq \bar{y}_{1} \text {. }
$$


Then the algorithm is true since we choose the smallest value of $y_{1}$ such that the constraint satisfies.

For $n=2$, we get the corresponding problem

$$
\min y_{1}+y_{2} \quad \text { s.t. } y_{1} \geq b_{1}, y_{1}+y_{2} \geq b_{2}, y_{2} \geq b_{3}, 0 \leq y_{1} \leq \bar{y}_{1}, 0 \leq y_{2} \leq \bar{y}_{2} .
$$

Without loss of generality, we can assume that $b_{1}, b_{2}, b_{3} \geq 0$. Otherwise, we replace $b_{i}<0$ by 0 for $i=1,2,3$. It is easy to observe that the optimal objective value of the problem is $b_{2}$ if $b_{1}+b_{3} \leq b_{2}$ or $b_{1}+b_{3}$ otherwise; see Figures 4.1 and 4.2 . In all cases, Algorithm 4.1 yields an optimal solution. Thus, our claim is true for the case $n=2$.

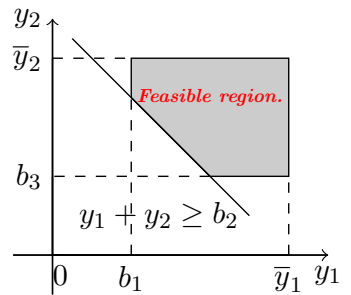

Figure 4.1: The feasible region in case $b_{1}+b_{3} \leq b_{2}$.

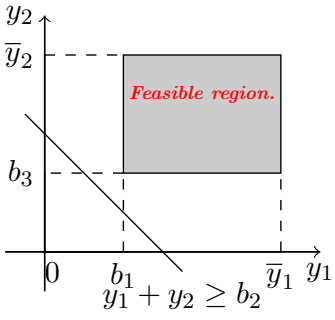

Figure 4.2: The feasible region in case $b_{1}+b_{3}>b_{2}$.

Now we consider the induction step. Assume that Algorithm 4.1 yields an optimal solution for the problem with $n-1$ variables, we further prove that it also yields an optimal solution for the problem with $n$ variables. We first fix a variable $y_{n}:=t \in\left[b_{n+1}, \bar{y}_{n}\right]$ and consider the problem with $n-1$ variables, i.e., the last row of the new problem is $y_{n-1} \geq b_{n}-t$. We further call this problem the induced problem. The optimal objective value of the original problem (with parameter $y_{n}:=t$ ) is the sum of the optimal objective value of the induced problem and $t$. By the induction step, we can apply Algorithm 4.1 to find the optimal solution of the induced problem.

Consider the original problem, we apply Algorithm 4.1 to the iteration $n-1$. Assume that we get the current variable values $y_{1}^{*}, y_{2}^{*}, \ldots, y_{n-1}^{*}$, there may exist the following cases:

1. If $y_{n-1}^{*}+\bar{y}_{n} \geq b_{n}$, we choose $y_{n}=t^{*}:=\max \left\{b_{n+1}, b_{n}-y_{n}^{*}\right\}$. By the induction step $\left\{y_{1}^{*}, y_{2}^{*}, \ldots, y_{n-1}^{*}\right\}$ is the optimal solution of the induced problem with a fixed parameter $y_{n}^{*}=t^{*}$. As $t^{*}$ is the minimum value such that the original problem is feasible, we obtain an optimal solution $\left(y_{1}^{*}, y_{2}^{*}, \ldots, y_{n-1}^{*}, y_{n}^{*}\right)$.

2. If $y_{n-1}^{*}+\bar{y}_{n}<b_{n}$, we fix $y_{n}:=\bar{y}_{n}$ and update the value of $y_{n-1}$ by $y_{n-1}^{*}:=b_{n}-\bar{y}_{n}$ as in Algorithm 4.1. Consider a new parameter $y_{n}:=\bar{y}_{n}-\delta \geq b_{n+1}$, we have to increase $y_{n-1}$ by $\delta$, i.e., $y_{n-1}:=y_{n-1}^{*}+\delta$. It holds $y_{n-1}^{*}+\delta \leq \bar{y}_{n-1}$, otherwise the problem is infeasible. Thus, the optimal objective value of the original problem does 
not change. In other words, we also get the optimal objective value in this case by applying Algorithm 4.1.

By induction, we imply that Algorithm 4.1 yields an optimal solution.

Based on the previous analysis, we get the following result.

Theorem 4.20. The uniform-cost inverse anti-3-centrum problem on cycles is solvable in linear time.

- The uniform-cost inverse 1-median problem on cycles.

Let us further consider $k=M$, the corresponding problem is the inverse 1-median problem. Moreover, the cost to modify one unit length of each edge is 1 , i.e., $c_{e}^{+}=c_{e}^{-}=1$. As usual, we classify the problem into two different cases:

Case 1. If $M=2 m+1$ is an odd integer, the elements of vector $\pi_{i}(a)-a$ hold the following property.

Lemma 4.21. Let $d^{i}=\pi_{i}(a)-a=\left(d_{1}^{i}, d_{2}^{i}, \ldots, d_{2 m+1}^{i}\right)$ for some $i \in\{1, \ldots, 2 m\}$ then we have

$$
d_{1}^{i} \leq d_{2}^{i} \leq \cdots \leq d_{m+1}^{i} \quad \text { and } \quad d_{2 m+1}^{i} \leq d_{2 m}^{i} \leq \cdots \leq d_{m+2}^{i}
$$

Proof. Assume that $1 \leq j \leq m$ we express $d_{j}^{i}$ and $d_{j+1}^{i}$ as follows:

$d_{j}^{i}=\left(\pi_{i}(a)-a\right)_{j}=\left(\pi_{i}(a)\right)_{j}-(m+1-j) \quad$ and $\quad d_{j+1}^{i}=\left(\pi_{i}(a)-a\right)_{j+1}=\left(\pi_{i}(a)\right)_{j+1}-(m-j)$.

We consider the two adjacent components of $\pi_{i}(a)$ in the following cases:

- If $\pi_{i}(a)_{j}=\pi_{i}(a)_{j+1}$, then $d_{j}^{i}<d_{j+1}^{i}$.

- If $\pi_{i}(a)_{j}=\pi_{i}(a)_{j+1}-1$, then $d_{j}^{i}=d_{j+1}^{i}$.

- If $\pi_{i}(a)_{j}=\pi_{i}(a)_{j+1}+1$, then $d_{j}^{i}<d_{j+1}^{i}$.

Thus, we get $d_{j}^{i} \leq d_{j+1}^{i}$ for $j=1,2, \ldots, m$. For the case $m+2 \leq j \leq 2 m$, we can prove correctness of the lemma by the same technique.

We consider new variables by the assignments

$$
\begin{aligned}
& x_{1}:=p_{m+1}, x_{2}:=p_{m}, \ldots, x_{m+1}:=p_{1}, \quad y_{1}:=p_{m+2}, y_{2}:=p_{m+1}, \ldots, y_{m}:=p_{2 m+1}, \\
& z_{1}:=q_{1}, z_{2}:=q_{2}, \ldots, z_{m+1}:=q_{m+1}, \quad t_{1}:=q_{2 m+1}, \ldots, t_{m-1}:=q_{m+3}, t_{m}:=q_{m+2} .
\end{aligned}
$$

The upper bounds of new variables are the bounds of the corresponding old ones, e.g., $\bar{x}_{1}=\bar{p}_{m+1}$ and so on. Using the new variables the inverse 1-median problem on $C$ can be formulated as the following linear programming problem. 
Proposition 4.22. Problem (4.4) can be rewritten as

$$
\begin{array}{ll}
\min & \sum_{j=1}^{m}\left(x_{j}+y_{j}+z_{j}+t_{j}\right)+x_{m+1}+z_{m+1} \\
\text { s.t. } & R x+R^{\prime} y+S z+S^{\prime} t \geq b, 0 \leq x \leq \bar{x}, 0 \leq y \leq \bar{y}, 0 \leq z \leq \bar{z}, 0 \leq t \leq \bar{t},
\end{array}
$$

where $R=\left(r_{i j}\right), R^{\prime}=\left(r_{i j}^{\prime}\right), S=\left(s_{i j}\right), S^{\prime}=\left(s_{i j}^{\prime}\right)$ and the corresponding coefficients are

$$
\begin{gathered}
r_{i j}=\left(\pi_{i}(a)-a\right)_{m+2-j} \text { for } j=1, \ldots, m+1, \quad r_{i j}^{\prime}=\left(\pi_{i}(a)-a\right)_{m+1+j} \text { for } j=1, \ldots, m, \\
s_{i j}=-\left(\pi_{i}(a)-a\right)_{j} \text { for } j=1, \ldots, m+1, \quad s_{i j}^{\prime}=-\left(\pi_{i}(a)-a\right)_{2 m+2-j} \text { for } j=1, \ldots, m .
\end{gathered}
$$

From Lemma 4.21, the coefficients of matrix $R, R^{\prime}, S, S^{\prime}$ in 4.9 satisfy

$$
\begin{gathered}
r_{i j} \geq r_{i j+1} \text { for } j=1, \ldots, m, \quad r_{i j}^{\prime} \geq r_{i j+1}^{\prime} \text { for } j=1, \ldots, m-1, \\
s_{i j} \geq s_{i j+1} \text { for } j=1, \ldots, m \text { and } s_{i j}^{\prime} \geq s_{i j+1}^{\prime} \text { for } j=1, \ldots, m-1 .
\end{gathered}
$$

If the edge lengths are only allowed to be increased or decreased the coefficients of 4.9 can be divided into two groups of nonincreasing coefficients. Therefore, the methods proposed by Burkard et al. [10] can be applied to solve 4.9 in $O\left(M^{2}\right)$ time.

Case 2. If $M$ is even, we get a similar result as for the case where $k$ is odd by using the same arguments.

In summary, we obtain the final result as follows.

Theorem 4.23. The uniform-cost inverse 1-median problem on cycles can be solved in $O\left(M^{2}\right)$ time, provided that the edge lengths are either increased or decreased.

\section{Conclusions}

We addressed the inverse anti- $k$-centrum problem on networks. We proved that the inverse anti- $k$-centrum problem is NP-hard even on tree networks. However, this problem on an unweighted cycle is polynomially solvable. Special cases with efficient algorithms were also discussed in details. Solution approaches for the inverse anti- $k$-centrum problem on cycles can be easily extended in order to get similar results for path graphs. Concerning future research, we can consider some other special cases of the inverse concave ordered 1-median problem on trees or cycles with polynomial time solvability. Additionally, the inverse ordered 1-median problem with variable vertex weights is also a promising topic.

\section{References}

[1] R. K. Ahuja, T. L. Magnanti and J. B. Orlin, Network Flows: Theory, algorithms, and applications, Prentice Hall, Englewood Cliffs, NJ, 1993. 
[2] B. Alizadeh and R. E. Burkard, Combinatorial algorithms for inverse absolute and vertex 1-center location problems on trees, Networks 58 (2011), no. 3, 190-200.

[3] _ Uniform-cost inverse absolute and vertex center location problems with edge length variations on trees, Discrete Appl. Math. 159 (2011), no. 8, 706-716.

[4] _ A linear time algorithm for inverse obnoxious center location problems on networks, CEJOR Cent. Eur. J. Oper. Res. 21 (2013), no. 3, 585-594.

[5] B. Alizadeh, R. E. Burkard and U. Pferschy, Inverse 1-center location problems with edge length augmentation on trees, Computing 86 (2009), no. 4, 331-343.

[6] F. B. Bonab, R. E. Burkard and B. Alizadeh, Inverse median location problems with variable coordinates, CEJOR Cent. Eur. J. Oper. Res. 18 (2010), no. 3, 365-381.

[7] F. B. Bonab, R. E. Burkard and E. Gassner, Inverse p-median problems with variable edge lengths, Math. Methods Oper. Res. 73 (2011), no. 2, 263-280.

[8] R. E. Burkard, M. Galavii and E. Gassner, The inverse Fermat-Weber problem, European J. Oper. Res. 206 (2010), no. 1, 11-17.

[9] R. E. Burkard, C. Pleschiutschnig and J. Zhang, Inverse median problems, Discrete Optim. 1 (2004), no. 1, 23-39.

[10] - The inverse 1-median problem on a cycle, Discrete Optim. 5 (2008), no. 2, $242-253$.

[11] M. C. Cai, X. G. Yang and J. Z. Zhang, The complexity analysis of the inverse center location problem, J. Global Optim. 15 (1999), no. 2, 213-218.

[12] Z. Drezner and H. W. Hamacher, Facility Location: Applications and theory, SpringerVerlag, Berlin, 2002.

[13] H. A. Eiselt and V. Marianov, Foundations of location analysis, International Series in Operations Research and Management Science, Springer, 2011.

[14] M. Galavii, The inverse 1-median problem on a tree and on a path, Electronic Notes in Disrete Mathematics 36 (2010), 1241-1248.

[15] M. R. Garey and D. S. Johnson, Computers and Intractability: A guide to the theory of NP-completeness, Series of Books in the Mathematical Sciences, W. H. Freeman, San Francisco, CA, 1979.

[16] E. Gassner, An inverse approach to convex ordered median problems in trees, J. Comb. Optim. 23 (2012), no. 2, 261-273. 
[17] A. J. Goldman, Optimal center location in simple networks, Transportation Sci. 5 (1971), 212-221.

[18] H. W. Hamacher, Mathematische Lösungsverfahren für planare Standortprobleme, Vieweg and Teubner Verlag, Wiesbaden, 1995.

[19] L. K. Hua, Application off mathematical models to wheat harvesting, Chinese Mathematics (1962), no. 2, 539-560.

[20] K. T. Nguyen, Inverse 1-median problem on block graphs with variable vertex weights, J. Optim. Theory Appl. 168 (2016), no. 3, 944-957.

[21] Some polynomially solvable cases of the inverse ordered 1-median problem on trees, Filomat 31 (2017), no. 12, 3651-3664.

[22] K. T. Nguyen and L. Q. Anh, Inverse $k$-centrum problem on trees with variable vertex weights, Math. Methods Oper. Res. 82 (2015), no. 1, 19-30.

[23] K. T. Nguyen and A. Chassein, The inverse convex ordered 1-median problem on trees under Chebyshev norm and Hamming distance, European J. Oper. Res. 247 (2015), no. $3,774-781$.

[24] K. T. Nguyen and A. R. Sepasian, The inverse 1-center problem on trees with variable edge lengths under Chebyshev norm and Hamming distance, J. Comb. Optim. 32 (2016), no. 3, 872-884.

[25] S. Nickel and J. Puerto, Location Theory: A unified approach, Berlin, Springer, 2005.

Van Huy Pham

AI Lab, Faculty of Information Technology, Ton Duc Thang University, Ho Chi Minh

City, Vietnam

E-mail address: phamvanhuy@tdtu.edu.vn

Kien Trung Nguyen

Department of Mathematics, Teacher College, Can Tho University, Can Tho, Vietnam

E-mail address: trungkien@ctu.edu.vn 